\title{
Stationäre Krankenhausbehandlung von Tuberkulosepatienten und Management der MDR-TB: Eine Arbeitshilfe für den ÖGD
}

Der Arbeitskreis Tuberkulose des Fachausschusses Infektionsschutz des Bundesverbandes der Ärztinnen und Ärzte des Öffentlichen Gesundheitsdienstes (BVÖGD) ist ein informelles Gremium von etwa 85 Ärztinnen und Ärzten, die zumeist in Gesundheitsämtern im Bereich der Tuberkulosefürsorge arbeiten, aber auch in Kliniken und Landesgesundheitsämtern. Der Arbeitskreis hat eine praxisorientierte Arbeitshilfe zur stationären Krankenhausbehandlung von Tuberkulosepatienten und zum Management der multiresistenten Tuberkulose (MDR-TB) erarbeitet. Das Papier wird in Kürze erscheinen.

Dieser Beitrag soll einen kurzen Überblick entlang häufig auftretender Fragen geben.

\section{Stationäre Behandlung von Tuberkulosepatienten}

\section{Wie lange müssen Tuberkulosepatienten stationär behan- delt werden? Welche Kriterien kann es geben?}

Für primär ansteckende Patienten (mikroskopischer oder nur kultureller Nachweis in respiratorischem Material) mit voll sensiblen Stämmen bzw. ohne anamnestischen Hinweis auf MDR-TB vor Vorliegen des Resistogramms sind folgende Bedingungen vor Beendigung der Isolierung $\mathrm{zu}$ fordern:

- drei mikroskopisch negative Sputen und

klinische und radiologische Besserung und

- Compliance des Patienten

Eine frühere Entlassung oder eine vollständig ambulante Behandlung ist bei Patienten ohne Risikofaktoren prinzipiell möglich, wenn die psychosozialen und häuslichen Rahmenbedingungen dies zulassen.

Für MDR-Patienten halten es die Autoren (über die Anforderungen gemäß DZK-Empfehlungen hinausgehend) für wichtig, vor Beendigung der Isolierung zusätzlich in der Regel drei negative Kulturen zu verlangen.

Medizinische Gründe für stationäre Krankenhausbehandlung im Sinne des $\int 39$ SGB V, Abs. I:

Allgemeine klinische Kriterien, die die Notwendigkeit einer initial stationären Behandlung auch bei vollsensiblen Tuberkulosen begründen, sind nach Ansicht des Arbeitskreises Tuberkulose:

1. Schweregrad der Tuberkulose (Ausdehnung, Symptome und Komplikationen)

2. Schwere Grund-/Begleitkrankheiten (z. B. auch bestimmte psychiatrische Erkrankungen wie Demenz oder Psychosen)

3. Therapieprobleme (Verdacht auf Mehrfachresistenz aufgrund von Vorbehandlung, Herkunftsland oder entsprechender Ansteckungsquelle, schwerwiegende unerwünschte Arzneimittelwirkungen, intravenöse Behandlung)

4. Mangelnde Mitarbeit (Compliance; insbesondere durch Alkoholkrankheit, schwierige psychosoziale Verhältnisse)

5. Ambulant nicht zu klärende Diagnose

6. Ungenügende Infrastruktur für eine ambulante Behandlung (z. B. zu geringe Facharztdichte)

Die Gründe sind durch die Klinik auch im Behandlungsverlauf sorgfältig zu dokumentieren.

Gründe des Infektionsschutzes im Sinne des $\int 30$ Abs. I Infektionsschutzgesetz (IfSG):

„Bei [...] Kranken sowie Krankheitsverdächtigen [...] kann angeordnet werden, dass sie in einem geeigneten Krankenhaus oder in sonst geeigneter Weise abgesondert werden."

Solange eindeutige medizinische Gründe für eine Krankenhausbehandlung bestehen, erübrigt sich in der Regel eine entsprechende Anordnung durch die zuständige Behörde. Ansteckungsfähigkeit allein ist nicht als medizinischer Grund im Sinne des SGB V anzusehen. Häufig geht sie jedoch mit dem klinischen Zustand einher, d.h. mit der Besserung des Zustandes geht meist auch die Ansteckungsfähigkeit zurück. Sobald medizinische Gründe nicht mehr vorliegen und eine Entlassung geplant ist, muss das Gesundheitsamt informiert werden, das dann über die Notwendigkeit einer weiteren Isolierung zu entscheiden hat.

Eine Isolierung aus Gründen des Infektionsschutzes kann im bereits behandelnden Krankenhaus erfolgen; es ist dann ein Tagessatz zur Unterbringung zu verhandeln, der die medizinischen Kosten nicht einschließt und der von der zuständigen Behörde zu tragen ist. Die medizinischen Kosten müssen weiterhin vom Träger der Krankenversicherung übernommen werden. Prinzipiell kommen auch andere Arten der Isolierung in Frage, typischerweise zu Hause, sofern die häuslichen und familiären Bedingungen dies zulassen.

\section{Management der multiresistenten Tuberkulose}

\section{Wie kann man eine ambulante Behandlung organisieren?}

In der Regel ist als Grundvoraussetzung für die ambulante Behandlung von Patienten mit MDR-TB oder anderen komplexen, schwierig $\mathrm{zu}$ behandelnden Tuberkulosen $\mathrm{zu}$ fordern, dass keine Ansteckungsfähigkeit mehr besteht. Zwingend notwendig ist eine Fallmanagerin/ein Fallmanager mit medizinischer oder sozialmedizinischer Ausbildung, die bzw. der die Behandlung koordiniert, engen Kontakt zum Patienten und $\mathrm{zu}$ allen beteiligten Akteuren hält und typischerweise an das Gesundheitsamt angebunden ist. Die Therapie einer MDR-TB gehört in die Hand erfahrener Fachärzte. In der Regel sind dies entweder niedergelassene 
Pneumologen, Infektiologen oder Pädiater oder entsprechende Fachärzte in Ambulanzen spezialisierter Fachkliniken.

\section{Sicherstellung der Medikamentengabe}

Prinzipiell wird in der Therapie der MDR-TB eine direkt überwachte Medikamentengabe (directly observed therapy, DOT) als Standard angesehen. Die überwachte Gabe oraler Medikamente sollte unter Aufsicht von geschultem Fachpersonal erfolgen. Dies ist zu Hause unter Einbeziehung ambulanter Pflegedienste möglich, in stationären Einrichtungen wie Pflegeheimen oder Obdachlosenheimen durch Pflegepersonal oder Sozialarbeiter, in Einzelfällen in Arztpraxen oder im Gesundheitsamt. Eine Herausforderung stellt die Organisation einer monatelangen, ambulanten intravenösen oder intramuskulären Therapie während der Kontinuitätsphase dar.

\section{Fazit}

Die Arbeitshilfe ist ein Beispiel dafür, wie der Arbeitskreis als aktives Diskussionsforum Fragen aus dem Berufsalltag aufgreift und Brücken zwischen Empfehlungen und ihrer praktischen Umsetzung baut. Sie dient der engen Kooperation zwischen Behandlern, Gesundheitsamt und anderen Akteuren und einer erfolgreichen Behandlung der Patienten auch in schwierigen Fällen.

\section{Wo finde ich Beratung und Unterstützung?}

- Für allgemeine, infektionsepidemiologische und das Meldewesen betreffende Fachinformationen zur Tuberkulose beim Robert Koch-Institut: www.rki.de/tuberkulose

Für Fragen des Fallmanagements, Umgebungsuntersuchungen etc. beim Arbeitskreis Tuberkulose im Fachausschuss Infektionsschutz des BVÖGD: www.rki.de/epidbull > Ausgabe 11/2002

- Für Therapiefragen zur MDR-TB, zu Laborfragen und zur Typisierung beim Forschungszentrum Borstel: www.fz-borstel.de

Für Therapiefragen beim DZK: www.dzk-tuberkulose.de

Für Fragen zur Tuberkulose bei Kindern bei der Deutschen Gesellschaft für pädiatrische Infektiologie (DGPI): http://dgpi.de

Die Empfehlungen sind verfügbar unter: www.dzk-tuberkulose.de/empfehlungen

- Dr. Martin Priwitzer*| Dr. Udo Götsch ** "Gesundheitsamt der Landeshauptstadt Stuttgart * Gesundheitsamt Frankfurt am Main Korrespondenz: Martin.Priwitzer@stuttgart.de

- Vorgeschlagene Zitierweise: Priwitzer M, Götsch U: Stationäre Krankenhausbehandlung von Tuberkulosepatienten und Management der MDR-TB: Eine Arbeitshilfe für den ÖGD. Epid Bull 2017;11/12:105- 106 DOI 10.17886/EpiBull-2017-014 